\title{
Interventions to Enhance Medication Safety in Residential Aged Care Settings: An Umbrella Review
}

\author{
Shadi Alruthea ${ }^{1}$, Paula Bowman ${ }^{2}$, Amina Tariq ${ }^{2}$, and Reece Hinchcliff ${ }^{2}$ \\ ${ }^{1}$ Qassim University \\ ${ }^{2}$ Queensland University of Technology Faculty of Health
}

June 23, 2021

\begin{abstract}
Aim: To conduct the first systematic synthesis of existing evidence reviews on interventions to enhance medication safety in RACS, to establish and compare their effectiveness. Method: This umbrella review included examination of meta-analyses, scoping and systematic reviews. Four electronic databases (MEDLINE, EMBASE, CINAHL, and The Cochrane library database of Systematic Reviews) were explored for eligible reviews. Those meeting the inclusion criteria were critically appraised using the JBI Critical Appraisal Instrument for Systematic reviews and Research Syntheses by two authors. Results: Fourteen reviews covering 166 unique, primary studies were included. Interventions were grouped according to type: medication review $(\mathrm{n}=12)$; staff education $(\mathrm{n}=8)$; multidisciplinary team meetings $(\mathrm{n}=6)$; computerised clinical decision support systems $(\mathrm{n}=$ $5)$; and transferring medicines information between health care settings $(n=1)$. Most reviews showed mixed evidence to support interventions' effectiveness, due to the significant heterogeneity between original research studies in respect to sites, samples sizes and intervention periods. However, in all intervention categories, pharmacists' collaboration with other health care professionals was most beneficial, showing definitive evidence for improving medication safety and quality of prescribing in RACS. The evidence suggests that combining two or more interventions is the most promising approach, despite this presenting implementation barriers in the resource-limited environments of many RACS, and methodological challenges in identifying the precise contribution of individual interventions, when implemented concurrently. Conclusion: Health stakeholders should explore a combination of at least two interventions, such as medication review and staff education, to improve medication safety in RACS.
\end{abstract}

\section{Hosted file}

Submitted Manuscript.docx available at https://authorea.com/users/421223/articles/527335interventions-to-enhance-medication-safety-in-residential-aged-care-settings-anumbrella-review 


\begin{tabular}{|c|c|}
\hline Element & Eligibility \\
\hline Study type & $\begin{array}{ll}\text { - } & \text { Systematic reviews } \\
\text { - } & \text { Scoping reviews } \\
- & \text { Meta-analyses }\end{array}$ \\
\hline Participants & $\begin{array}{ll}- & \text { Residential aged care facilities } \\
- & \text { Nursing homes } \\
- & \text { Long-term care facilities } \\
- & \text { Care homes } \\
\text { - } & \text { Skilled nursing facilities }\end{array}$ \\
\hline Intervention & $\begin{array}{ll}\text { - } & \text { Interventions } \\
\text { - } & \text { Methods } \\
\text { - } & \text { Policies } \\
\text { - } & \text { Strategies } \\
\text { - } & \text { Programs }\end{array}$ \\
\hline Outcome & $\begin{array}{ll}\text { - } & \text { Reducing or preventing medication errors } \\
\text { - } & \text { Enhancing medication safety } \\
\text { - } & \text { Medication appropriateness } \\
\text { Quality use of medications }\end{array}$ \\
\hline
\end{tabular}




\begin{tabular}{|c|c|}
\hline \multicolumn{2}{|r|}{ Search Strategy } \\
\hline Concept & Search Terms \\
\hline Aged Care Facility & $\begin{array}{l}\text { Aged care OR Nursing home* OR Residential aged care OR } \\
\text { Residential care OR Long-term care }\end{array}$ \\
\hline Intervention & Intervention* OR Strateg* OR Polic* OR Program* OR Method* \\
\hline Medication & Medication OR Adverse event ${ }^{\star}$ OR Medication error ${ }^{\star}$ \\
\hline
\end{tabular}



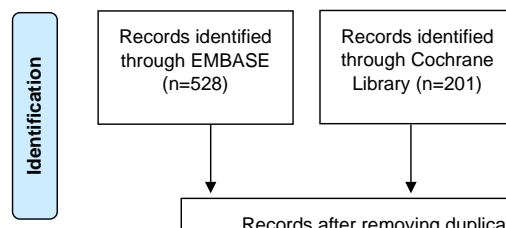

Records identified Records identified
through MEDLINE

Library $(n=201)$

through CINA
$(\mathrm{n}=171)$

$(n=160)$

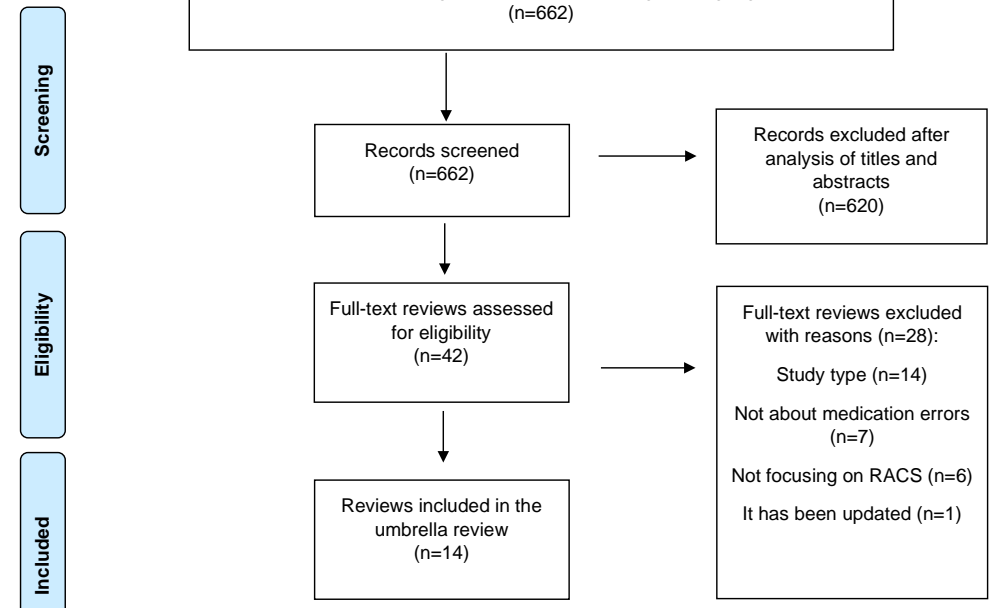

Records after removing duplicates and non-English language studies $(n=662)$

\section{Hosted file}

Table 03.pdf available at https://authorea.com/users/421223/articles/527335-interventions-toenhance-medication-safety-in-residential-aged-care-settings-an-umbrella-review 


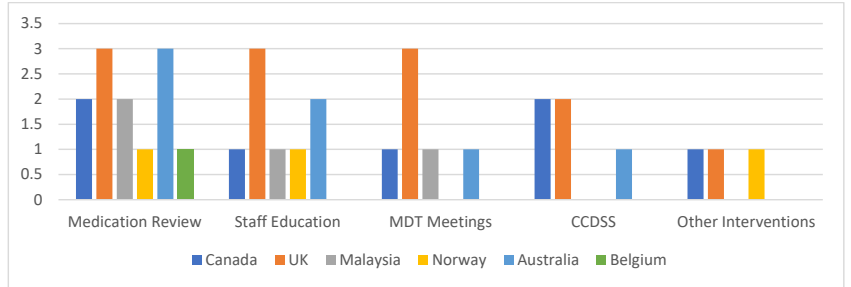

\section{Hosted file}

Table 04.pdf available at https://authorea.com/users/421223/articles/527335-interventions-toenhance-medication-safety-in-residential-aged-care-settings-an-umbrella-review 


\begin{tabular}{|c|c|c|c|c|}
\hline & & $\begin{array}{l}\text { Categories of } \\
\text { Interventions }\end{array}$ & & \\
\hline $\begin{array}{l}\text { Medication } \\
\text { Review } \\
\text { (10 Reviews) }\end{array}$ & $\begin{array}{l}\text { Staff Education } \\
\text { (6 Reviews) }\end{array}$ & $\begin{array}{l}\text { Multidisciplinary } \\
\text { Team Meetings } \\
\text { (5 Reviews) }\end{array}$ & $\begin{array}{l}\text { Computerised } \\
\text { Clinical Decision } \\
\text { Support Systems } \\
\text { (4 Reviews) }\end{array}$ & $\begin{array}{l}\text { Other } \\
\text { Interventions } \\
\text { (3 Reviews) }\end{array}$ \\
\hline
\end{tabular}

\section{Hosted file}

Table 05.pdf available at https://authorea.com/users/421223/articles/527335-interventions-toenhance-medication-safety-in-residential-aged-care-settings-an-umbrella-review 